\title{
Protocol directed sedation by nurses reduced the duration of mechanical ventilation in critically ill patients
}

Brook AD, Ahrens TS, Schaiff R, et al. Effect of a nursing-implemented sedation protocol on the duration of mechanical ventilation. Crit Care Med 1999 Dec;27:2609-15.

QUESTION: Is protocol directed sedation by nurses associated with a shorter duration of mechanical ventilation than traditional, non-protocol directed sedation in critically ill patients with acute respiratory failure?

\section{Design}

Randomised (allocation concealed), unblinded, controlled trial.

\section{Setting}

A medical intensive care unit (ICU) in a university teaching hospital in St Louis, Missouri, USA.

\section{Patients}

321 patients $>17$ years of age (mean age 58 y, $51 \%$ women, $58 \%$ white) who required mechanical ventilation for acute respiratory failure. Patients were excluded if they were temporarily admitted to the medical ICU and awaiting admission to a surgical ICU.

\section{Intervention}

At the time of mechanical ventilation, patients were allocated to 1 of 2 groups. 162 patients received protocol directed sedation by nurses, in which nurses determined if analgesics, sedatives, or both were needed; type of administration (intravenous bolus or continuous intravenous infusion); and dosage. 159 patients received traditional, non-protocol directed sedation, in which physicians made all the sedation orders. All patients received protocol directed weaning from mechanical ventilation by nurses and respiratory therapists.

\section{Main outcome measures}

Duration of mechanical ventilation, ICU length of stay, hospital length of stay, hospital mortality, acquired organ system derangement, reintubation, and tracheostomy.

\section{Main results}

Analysis was by intention to treat. Protocol directed sedation compared with non-protocol directed sedation decreased the mean duration of mechanical ventilation (89 $v 124 \mathrm{~h}, \mathrm{p}=0.003)$, mean ICU length of stay (5.7 v $7.5 \mathrm{~d}, \mathrm{p}=0.013)$, mean hospital length of stay $(14 v 20 \mathrm{~d}$, $\mathrm{p}<0.001)$, and rate of tracheostomy $(6 \% v 13 \%$, $\mathrm{p}=0.038)$. No differences existed for hospital mortality $(30 \% v 36 \%, \mathrm{p}=0.3)$, mean number of acquired organ system derangements $(2.8 v 2.9, \mathrm{p}=0.7)$, or rate of reintubation $(9 \% \approx 13 \%, \mathrm{p}=0.2)$.

Source of funding. Innovations in Healthcare Program

For correspondence: Dr A D Brook, 10004 Kennerly Road, Suite 310A, St Louis, MO 63128, USA. Fax +1 3148498789 .

\section{Conclusion}

Protocol directed sedation by nurses reduced the duration of mechanical ventilation, the intensive care unit and hospital lengths of stay, and the need for tracheostomy compared with traditional non-protocol directed sedation in critically ill patients with acute respiratory failure.

\section{COMMENTARY}

Few studies are available on protocol directed sedation management in the ICU. The findings of this study by Brook et al support previous studies that have reported successful nurse managed sedation protocol use with intubated patients. $^{1}$

This is one of the first studies, however, to use a randomised controlled trial to address this question. The study is further strengthened by concealment of patient allocation through the use of opaque, sealed envelopes that were opened at the time each patient was enrolled and through the calculation of a sample size that ensured sufficient power. At the same time, the study had a number of limitations. Firstly, all patients were from one 19 bed, medical ICU and may not be representative of patients receiving mechanical ventilation in other ICUs; secondly, because the nurses and physicians managing sedation could not be blinded, it is possible that they may have altered their practices, especially the physician control group; thirdly, they did not examine the effect of sedation on sedation related outcomes, such as unplanned extubations and nosocomial pneumonia; and fourthly, they did not measure nurse compliance with the sedation protocol.

Sedation is an important part of the care of critically ill patients, especially those on mechanical ventilation. This study showed that ICU nurses, using a multidisciplinary designed sedation protocol, could safely administer and titrate intravenous sedation for critically ill patients. Although they did not conduct a formal economic analysis, the authors found that the shortened length of ICU and hospital stays and the reduced need for tracheostomy resulted in substantial cost savings. In ICUs where physicians are not constantly at the bedside, protocol directed sedation by nurses allows for more rapid clinical decision making at the bedside by eliminating the need for physician orders and thereby reducing the time needed to implement sedation changes. When considering implementation of this protocol in another hospital ICU setting, it would be important to keep in mind the level of physician support for nurse managed treatment using protocols, nursing staff willingness to accept the responsibility and autonomy, cost of training nursing staff, and decision making support mechanisms for the nursing staff.

Loris A Thomas, RN, ARNP, CS, CCRN, MS Acute Care Nurse Practitioner Clinical Assistant Professor University of Florida College of Nursing Gainesville, Florida, USA

1 Edwards D, Hess L. Aggressive weaning in cardiac surgical patients. Dimens Crit Care Nurs 1996;15:181-6.

2 Powers J. A sedation protocol for preventing patient self-extubation. Dimens Crit Care Nurs 1999;18:30-4 